\title{
Factors related to nausea/vomiting in lung cancer patients receiving chemotherapy
}

\author{
Chi-Hsiang Shen ${ }^{1}$, Li-Yu Yang ${ }^{* 2}$ \\ ${ }^{1}$ Department of Nursing, Kaohsiung Medical University Hospital, Kaohsiung Medical University, Taiwan \\ ${ }^{2}$ College of Nursing, Kaohsiung Medical University, Taiwan
}

Received: February 10, 2015

Accepted: April 2, 2015

Online Published: April 7, 2015

DOI: $10.5430 /$ cns.v3n3p27

URL: http://dx.doi.org/10.5430/cns.v3n3p27

\begin{abstract}
Background: The incidence and severity of Chemotherapy-induced nausea and vomiting (CINV) vary from patient to patient and are dependent on the emetogenicity of chemotherapy agents. Understanding of the correlations between demographic characteristics and nausea/vomiting, nurses could provide early nursing interventions for preventing CINV, thereby alleviating patients' discomfort.

Methods: A cross-sectional, correlational design was used. The purpose of this study was to understand the level of nausea/vomiting and related factors in lung cancer patients receiving chemotherapy and to determine the correlations between demographic characteristics and nausea/vomiting. The participants were a total of 70 lung cancer patients who had received at least one cycle of Cisplatin $\left(50-75 \mathrm{mg} / \mathrm{m}^{2}\right)$ and had previous experience of nausea/vomiting during chemotherapy. A demographic data sheet and the Morrow Assessment of Nausea and Emesis were used to collect and monitor participants' level of nausea and vomiting.

Results: Among the lung cancer patients receiving chemotherapy, $61.4 \%$ had moderate to severe nausea; $28.6 \%$ had mild to severe vomiting; $72.9 \%$ reported that the feeling of nausea lasted for 2 to 5 hours; $51.4 \%$ stated that the most severe nausea occurred during 12 and 24 hours after receiving chemotherapy. The patients with previous experience of motion sickness had higher level of nausea during chemotherapy $(p=.036)$; the patients who received the sixth or more cycles of chemotherapy had higher level of vomiting during chemotherapy $(p=.010)$; the patients who had higher level of nausea had higher frequency of additional use of antiemetics $(p<.0001)$.

Conclusions: The results showed the patients with previous experience of motion sickness or those with additional use of antiemetics were likely to have higher level of nausea. Chemotherapy cycles were correlated with patients' level of vomiting. Therefore, nurses should provide appropriate pre-chemotherapy nursing interventions for lung cancer patients with previous experience of motion sickness or those receiving the sixth or more cycles of chemotherapy to prevent the occurrence of nausea and vomiting.
\end{abstract}

Key Words: Lung cancer patients, Nausea, Vomiting

\section{INTRODUCTION}

Chemotherapy is recommended as one of primary treatments for lung cancer. According to the National Comprehensive Cancer Network (NCCN) guidelines, ${ }^{[1]}$ platinum-based chemotherapy (Cisplatin or Carboplatin) in combination with other commonly used chemotherapy agents, such as Paclitaxel (Taxol), Docetaxel (Taxotere), Vinorelbine (Navelbine), Gemcitabine (Gemzar), Pemetrexed (Alimta) and Etopo-

\footnotetext{
*Correspondence: Li-Yu Yang; Email: a885019@kmu.edu.tw; Address: College of Nursing, Kaohsiung Medical University, No.100, Shih-Chuan 1st Road, Kaohsiung 80756, Taiwan.
} 
side (Vepesid) is recommended as a primary treatment for advanced lung cancer patients who cannot have surgery. Chemotherapy agents kill fast-growing cancer cells and prolong life. However, they also damage some normal cells, resulting in many side effects, of which nausea and vomiting are the most common, with an incidence of over $90 \%$ of patients without antiemetic prophylaxis. ${ }^{[2,3]}$ Nausea and vomiting not only cause fluid and electrolyte imbalance as well as reduced physical activity but also affect patients' quality of life and reduced interpersonal relationships, lead to malnutrition, dehydration, weakness and fatigue, thereby reducing patients' willingness to continue to receive treatment. In serious cases, they may lead to death due to complications. ${ }^{[4]}$

Chemotherapy-induced nausea and vomiting (CINV) are primarily regulated by chemoreceptor trigger zone (CTZ) and vomiting center. ${ }^{[5]}$ Emetogenic chemotherapy, or its metabolites stimulate the CTZ located in the floor of the fourth ventricle through bloodstream or cerebrospinal fluid, leading to the release of emetic neurotransmitters including Serotonin (5-HT), Substance P (SP), and Dopamine $\left(\mathrm{D}_{2}\right)$, which in turn activates the vomiting center in medulla to produce vomiting. ${ }^{[5,6]}$ In addition, association of previous knowledge and experience or previous experience of chemotherapy activates the cerebral cortex to produce anticipatory vomiting through the release of neurotransmitters including gammaaminobutyric acid (GABA) and histamine. ${ }^{[6,7]}$ Moreover, the incidence and severity of chemotherapy-induced nausea and vomiting vary from patient to patient and are dependent on the emetogenicity of chemotherapy agents. ${ }^{[2,5]}$ According to the American Society of Clinical Oncology (ASCO) and NCCN guidelines, ${ }^{[2,3,8]}$ the emetogenicity scale have divided into the following 4 categories: highly emetogenic agents and that cause CINV in greater than $90 \%$ of patients (e.g., Cisplatin), moderate emetogenic agents and that induce CINV in $30 \%$ to $90 \%$ of patients (e.g., Carboplatin), low emetogenic agents and that are associated with CINV rates of $10 \%$ to $30 \%$ (e.g., Docetaxel, Etoposide, \& Gemcitabine), and minimally emetogenic agents and that cause CINV in less than $10 \%$ of patients (e.g., Vinorelbine).

Chemotherapy is recommended as one of primary treatments for lung cancer. CINV are the most common side effects of chemotherapy treatment. Previous studies pointed out the using routine antiemetic regimens in patients receiving chemotherapy remain experienced nausea and vomiting in approximately 39\%-55\%. ${ }^{[9,10]}$ Some Western studies have found that CINV were associated with gender, age, and life experiences. ${ }^{[7,11,12]}$ In a prospective, multicenter and observational study was enrolled 277 patients, female patients and younger patients reported significantly more CINV than male and older patients. ${ }^{[10]}$ Furthermore, a single-institution, prospective, observational study was enrolled 108 patients, history of motion sickness was associated with delayed vomiting. ${ }^{[13]}$ However, domestic research is not available on the correlations between demographic characteristics and CINV. An in-depth understanding of the correlations between demographic characteristics and CINV is necessary, which could help understand the differences between Eastern and Western people and provide lung cancer patients who receive chemotherapy with early preventive nursing measures, thereby alleviating patient discomfort and elevating these patients' willingness to receive treatment. Therefore, the purpose of this study was to explore nausea and vomiting experiences in lung cancer patients receiving chemotherapy and the correlations between demographic characteristics and nausea/vomiting experiences in lung cancer patients receiving chemotherapy. This study was expected to serve as a reference in caring for lung cancer patients during chemotherapy.

\section{METHODS}

\subsection{Study design}

A cross-sectional and correlational design was used. From July 20 to December 31 in 2012, a total of 70 lung cancer patients who had received at least one cycle of Cisplatin $\left(50-75 \mathrm{mg} / \mathrm{m}^{2}\right)$ and intended to receive Cisplatin (50$75 \mathrm{mg} / \mathrm{m}^{2}$ ) in this hospitalization were recruited from the chest medicine ward of a medical center located in southern Taiwan. As recommended by the ASCO, all patients were given three antiemetics, including 5-Hydroxytryptamine 3 (5- $\left.\mathrm{HT}_{3}\right)$ serotonin receptor antagonist (Ramosetron), NK1 receptor antagonist (Aprepitant), and Dexamethasone during chemotherapy.

\subsection{Study questions}

The study questions were to understand the level of nausea/vomiting and related factors in lung cancer patients receiving chemotherapy and to determine the correlations between demographic characteristics and nausea/vomiting.

\subsection{Sample size}

The sample size was determined based on the table proposed by Polit and Beck ${ }^{[14]}$ for determining the sample size required to estimate the population correlation. A sample size of 70 was recommended to achieve a level of power of .8 and a medium effect size of .35 at a significant $\alpha$ level of .05 .

\subsection{Ethical considerations}

Patients were included in the study after giving informed consent. This study was reviewed and approved by the institutional review boards (KMUH-IRB-20120114). 


\subsection{Data collection and procedure}

The patients who met the inclusion criteria were explained about the purpose and procedure of this study and their rights. Then, the patients who agreed to participate in this study were asked to sign a consent form and complete a questionnaire. Inclusion criteria were as follows: (1) having received at least one cycle of Cisplatin $\left(50-75 \mathrm{mg} / \mathrm{m}^{2}\right)$ and intending to receive Cisplatin $\left(50-75 \mathrm{mg} / \mathrm{m}^{2}\right),(2)$ having previous experience of nausea/vomiting during chemotherapy, and (3) being conscious. Exclusion criteria included those who were unable to express themselves and those who were too physically weak. Total of 82 lung cancer patients were approached in this study and then 12 lung cancer patients were excluded.

\subsection{Measurements}

A structured questionnaire was used for data collection. The Morrow Assessment of Nausea and Emesis (MANE), a 16item self-administered scale, consists of 3 domains: frequency and severity of nausea/vomiting ( 2 items, rated on a 6 -point scale ranging from "very mild" to "intolerable"); the time and duration during which nausea/vomiting are worst (2 items, rated on a 7-point scale from "during chemotherapy" to "after chemotherapy"); and patients' subjective perception of the effects of antiemetics (1 item, rated on a 4-point scale ranging from "very effective" to "ineffective"). ${ }^{[15]}$ The mean test-retest reliability coefficients were calculated across four consecutive treatments and ranged from .72 to .96 , indicating acceptable reliability. ${ }^{[16]}$ The Cronbach's $\alpha$ of this study was .889 , indicating good internal consistency. The MANE is a simple cost-effective tool that allows researchers to comprehensively assess the frequency, severity, and duration of chemotherapy-related nausea and vomiting. The MANE scale permission was obtained from original author.

The self-made demographic questionnaire was based on literature review and composed of 9 items, including age, gender, marital status, education, past history (e.g., hypertension, heart failure, diabetes mellitus, gastrointestinal disease and kidney disease), previous experience of motion sickness, previous experience of alcohol drinking, number of chemotherapy cycle, and additional use of antiemetics.

\subsection{Data analysis}

Data were analyzed using SPSS 17.0 for Windows. Frequency distribution and percentage were used to analyze participants' demographic data and severity of nausea/vomiting. An independent samples $t$-test and one-way analysis of variance (One-way ANOVA) were used to analyze the correlations between demographic variables and level of nausea/vomiting.

Published by Sciedu Press
Table 1. Demographic characteristics $(\mathrm{N}=70)$

\begin{tabular}{|c|c|c|}
\hline Variables & n & $\%$ \\
\hline \multicolumn{3}{|l|}{ Age (years) } \\
\hline$\leqq 35$ & 3 & 4.3 \\
\hline $36-55$ & 18 & 25.7 \\
\hline $56-75$ & 49 & 70.0 \\
\hline \multicolumn{3}{|l|}{ Gender } \\
\hline Male & 43 & 61.4 \\
\hline Female & 27 & 38.6 \\
\hline \multicolumn{3}{|l|}{ Marital status } \\
\hline Single & 8 & 11.4 \\
\hline Married & 62 & 88.6 \\
\hline \multicolumn{3}{|l|}{ Education } \\
\hline$\leqq$ Elementary & 19 & 27.1 \\
\hline Junior high & 30 & 42.9 \\
\hline$\geqq$ Senior high & 21 & 30.0 \\
\hline \multicolumn{3}{|l|}{ Past history } \\
\hline 0 & 34 & 48.6 \\
\hline 1 disease & 14 & 20.0 \\
\hline$\geqq 2$ diseases & 22 & 31.4 \\
\hline \multicolumn{3}{|c|}{ Previous experience of motion sickness } \\
\hline No & 54 & 77.1 \\
\hline Yes & 16 & 22.9 \\
\hline \multicolumn{3}{|c|}{ Previous experience of alcohol drinking } \\
\hline No & 39 & 55.7 \\
\hline Yes & 31 & 44.3 \\
\hline \multicolumn{3}{|c|}{ Number of chemotherapy cycle } \\
\hline 2nd to 3rd & 36 & 51.4 \\
\hline 4th to 5 th & 16 & 22.9 \\
\hline 6th or more & 18 & 25.7 \\
\hline \multicolumn{3}{|c|}{ Additional use of antiemetics } \\
\hline No & 67 & 95.7 \\
\hline Yes & 3 & 4.3 \\
\hline
\end{tabular}

\section{Results}

\subsection{Participant characteristics}

The demographics for the 70 patients are summarized in Table 1. The mean age was $58.6( \pm 9.1)$ years, with a range of 33 to 74 years; most ( $n=49,70 \%)$ were at the age of 56 to 75 . Males were the majority ( $n=43,61.4 \%)$; most were married $(n=62,88.6 \%)$; most had an education level at junior high school ( $n=30,42.9 \%) ; 48.6 \%$ of the patients had no past history $(n=34)$; most had no previous experience of motion sickness $(n=54,77.1 \%)$; most had no previous experience of alcohol drinking $(n=39,55.7 \%) ; 51.4 \%$ of the patients received the second to the third cycle of chemotherapy ( $n$ $=36)$; most had no additional use of antiemetics $(n=67$, $95.7 \%)$. 


\subsection{Status quo analysis of nausea/vomiting in lung can-} cer patients receiving chemotherapy

Among the lung cancer patients receiving chemotherapy, most ( $n=43,61.4 \%$ ) had moderate to severe nausea; 50 patients $(71.4 \%$ ) did not experience vomiting (see Table 2). Further analysis found that 51 patients $(72.9 \%)$ had a feeling of nausea for 2 to 5 hours and that 36 patients (51.4\%) reported that the most severe nausea occurred during 12 and 24 hours after receiving chemotherapy.

Table 2. Status quo of nausea and vomiting $(\mathrm{N}=70)$

\begin{tabular}{lll}
\hline Variables & $\mathbf{n}$ & $\mathbf{\%}$ \\
\hline Level of nausea & & \\
$\quad$ None & 0 & 0.0 \\
$\quad$ Very mild to mild & 27 & 38.6 \\
$\quad$ Moderate to severe & 43 & 61.4 \\
Level of vomiting & & \\
$\quad$ None & 50 & 71.4 \\
$\quad$ Very mild to mild & 9 & 12.9 \\
$\quad$ Moderate to severe & 11 & 15.7 \\
\hline
\end{tabular}

3.3 Correlations of nausea/vomiting with demographic variables and disease characteristics in lung cancer patients receiving chemotherapy

The results of independent samples $t$-test and one-way analysis showed that the patients with previous experience of motion sickness had higher level of nausea than those with no such experience $(t=2.136, p=.036)$. Significant differences were found between cycle of chemotherapy and level of nausea/vomiting $(\mathrm{F}=4.96, p=.010)$; the patients receiving the sixth or more cycles of chemotherapy had higher level of vomiting than those receiving the second to the fifth cycle. The patients who had higher level of nausea had higher frequency of additional use of antiemetics $(t=11.09, p<.0001)$. No significant differences were found in other demographic variables (see Tables 3 and 4).

\section{Discussion}

CINV are the most common side effects of chemotherapy treatment. In this study, $61.4 \%$ and $28.6 \%$ of the patients experienced moderate to severe nausea and mild to severe vomiting, respectively. This finding is similar to the study of Celio et al. ${ }^{[9]}$ the use of antiemetics such as $5-\mathrm{HT}_{3}$ serotonin receptor antagonist (Palonosetron) and Dexamethasone still experienced vomiting in approximately $40 \%-50 \%$ of cancer patients. Therefore, patients not only need suggestions to use three antiemetics, including $5-\mathrm{HT}_{3}$ serotonin receptor antagonist, $\mathrm{NK}_{1}$ receptor antagonist, and Dexamethasone recommended by the ASCO during chemotherapy but also add non-pharmacologic interventions such as mas- sage or acupuncture and develop the clinical guidelines for non-pharmacologic interventions related to CINV to enhance the quality of care for cancer patients. ${ }^{[2,17,18]}$

Moreover, the results of this study showed that $72.9 \%$ of the patients had a feeling of nausea for 2 to 5 hours and that $51.4 \%$ of the patients reported that the most severe nausea occurred during 12 and 24 hours after receiving chemotherapy. Our findings are consistent with those of other studies, in which although antiemetics get better, however CINV remains an important issue for cancer patients. ${ }^{[10,19,20]}$ These articles may support the argument that most of the currently used antiemetics have better effects on vomiting and less effects on nausea. At present, the nursing care of nausea is primarily focused on health education to guide patients to apply frequent small meals, attention diversion, or deep breathing to alleviate their symptoms. Therefore, we suggest that individuation nursing interventions (e.g., complementary and alternative medicine, change lifestyle or diet habits) be provided in patients with CINV. ${ }^{[18,21]}$

The CINV were associated with some risk factors, such as age, gender, alcohol use, history of motion sickness and life experiences. ${ }^{[5,10,11,13]}$ In our study, no direct correlations of CINV with age, gender, marital status, past history, education level, previous experience of alcohol drinking. It is unclear why this is the case. The reason for this finding may be due to the constitutional differences between Eastern and Western people. According to the traditional Chinese medicine theory, the constitution will affect individual susceptibility to disease and response to drugs. ${ }^{[22,23]}$ Thus, it would be of interest to determine this issue.

Furthermore, we found that patients with previous experience of motion sickness had higher level of nausea than those with no such experience. This finding is congruent with the studies of Janelsin et al. ${ }^{[5]}$ and Shih, Wan, \& Chan. ${ }^{[13]}$ It may be inferred that patients with previous experience of motion sickness are likely to experience chemotherapy-related nausea. Therefore, we suggest that antiemetics and nursing strategies be used in patients with previous experience of motion sickness before the patients receiving chemotherapy.

Additionally, the current study found that the patients receiving the sixth or more cycles of chemotherapy had higher level of vomiting than those receiving the second to the fifth cycle. Therefore, we suggest increasing the use of antiemetics in patients receiving the sixth or more cycles of chemotherapy. Evidence to support the use of additional antiemetics during chemotherapy could reduce nausea/vomiting. ${ }^{[10,20]}$ Previous studies have pointed out that in addition to antiemetics, nonpharmacological interventions such as acupressure, massage, music therapy or relaxation can be utilized to improve self- 
care and reduce nausea/vomiting. ${ }^{[18]}$ Future research can determine the nursing strategies other than pharmacological explore the effects of interventions (acupressure, massage, interventions. relaxation) on nausea/vomiting in cancer patients to better

Table 3. Comparison analysis of demographic variables and level of nausea $(\mathrm{N}=70)$

\begin{tabular}{|c|c|c|c|c|c|}
\hline \multirow{2}{*}{ Variables } & \multirow{2}{*}{$\mathbf{n}$} & \multicolumn{4}{|c|}{ Level of nausea } \\
\hline & & $\mathbf{M} \pm$ SD & F/t & $p$ & LSD \\
\hline Age & & & 0.98 & $.380^{*}$ & \\
\hline Under 35 years & 3 & $3.33 \pm 1.15$ & & & \\
\hline 36-55 years & 18 & $2.72 \pm 0.96$ & & & \\
\hline $56-75$ years & 49 & $2.98 \pm 0.78$ & & & \\
\hline Gender & & & -0.27 & $.788^{\#}$ & \\
\hline Male & 43 & $2.91 \pm 0.87$ & & & \\
\hline Female & 27 & $2.96 \pm 0.81$ & & & \\
\hline Marital status & & & 0.25 & $.800^{\#}$ & \\
\hline Single & 8 & $3.00 \pm 0.93$ & & & \\
\hline Married & 62 & $2.92 \pm 0.84$ & & & \\
\hline Education & & & 0.14 & $.868^{*}$ & \\
\hline Elementary or below & 19 & $3.00 \pm 0.88$ & & & \\
\hline Junior high & 30 & $2.93 \pm 0.87$ & & & \\
\hline Senior high or above & 21 & $2.86 \pm 0.93$ & & & \\
\hline Past history & & & 0.66 & $.521^{*}$ & \\
\hline 0 & 34 & $2.88 \pm 0.84$ & & & \\
\hline 1 disease & 14 & $2.79 \pm 0.89$ & & & \\
\hline$\geqq 2$ diseases & 22 & $3.09 \pm 0.81$ & & & \\
\hline Previous experience of motion sickness & & & 2.14 & $.036^{\#}$ & \\
\hline No & 54 & $2.81 \pm 0.83$ & & & \\
\hline Yes & 16 & $3.31 \pm 0.79$ & & & \\
\hline Previous experience of alcohol drinking & & & -0.49 & $.620^{\#}$ & \\
\hline No & 39 & $2.97 \pm 0.78$ & & & \\
\hline Yes & 31 & $2.87 \pm 0.92$ & & & \\
\hline Number of chemotherapy cycle & & & 0.23 & $.792^{*}$ & \\
\hline Second to third ${ }^{1}$ & 36 & $2.86 \pm 0.80$ & & & \\
\hline Fourth to fifth ${ }^{2}$ & 16 & $3.0 \pm 0.89$ & & & \\
\hline Sixth or more ${ }^{3}$ & 18 & $3.0 \pm 0.91$ & & & \\
\hline Additional use of antiemetics & & & 11.09 & $<.0001^{\#}$ & \\
\hline No & 67 & $2.88 \pm 0.83$ & & & \\
\hline Yes & 3 & $4.0 \pm 0.0$ & & & \\
\hline
\end{tabular}

* Analysis was performed using One-way ANOVA; ${ }^{*}$ Analysis was performed using independent samples $t$-test

\section{Conclusions}

Overall, $72.9 \%$ of the lung cancer patients receiving chemotherapy had a feeling of nausea for 2 to 5 hours and $51.4 \%$ of those reported that the most severe nausea occurred during 12 and 24 hours after receiving chemotherapy. The findings suggest that most of the currently used antiemetics have better effects on vomiting and less effects on nausea. Moreover, the patients with previous experience of motion sickness or those with additional use of antiemetics had higher level of nausea. The patients receiving the sixth or more cycles of chemotherapy had higher level of vomit-

Published by Sciedu Press ing than those receiving the second to the fifth cycle, which suggests that the more chemotherapy cycles the patients receive, the greater the vomiting severity they have. Therefore, patients not only need suggestions to use three antiemetics, including 5- $\mathrm{HT}_{3}$ serotonin receptor antagonist, $\mathrm{NK}_{1}$ receptor antagonist, and Dexamethasone recommended by the ASCO during chemotherapy but also add non-pharmacologic interventions such as massage or acupuncture, and we need to develop the clinical guidelines for non-pharmacologic interventions related to CINV to enhance the quality of care for cancer patients. 
Table 4. Comparison analysis of demographic variables and level of vomiting $(\mathrm{N}=70)$

\begin{tabular}{|c|c|c|c|c|c|}
\hline \multirow{2}{*}{ Variables } & \multirow{2}{*}{$\mathbf{n}$} & \multicolumn{4}{|c|}{ Level of vomiting } \\
\hline & & M \pm SD & $\mathbf{F} / \mathbf{t}$ & $p$ & LSD \\
\hline Age & & & 1.78 & $.176^{*}$ & \\
\hline Under 35 years & 3 & $2.00 \pm 0.00$ & & & \\
\hline $36-55$ years & 18 & $0.78 \pm 1.35$ & & & \\
\hline 56-75 years & 49 & $0.63 \pm 1.20$ & & & \\
\hline Gender & & & 0.75 & $.471^{\#}$ & \\
\hline Male & 43 & $0.81 \pm 1.26$ & & & \\
\hline Female & 27 & $0.59 \pm 1.22$ & & & \\
\hline Marital status & & & 0.52 & $.959^{\#}$ & \\
\hline Single & 8 & $0.75 \pm 1.04$ & & & \\
\hline Married & 62 & $0.73 \pm 1.27$ & & & \\
\hline Education & & & 1.98 & $.146^{*}$ & \\
\hline Elementary or below & 19 & $0.95 \pm 1.35$ & & & \\
\hline Junior high & 30 & $0.90 \pm 1.30$ & & & \\
\hline Senior high or above & 21 & $0.29 \pm 0.96$ & & & \\
\hline Past history & & & 1.17 & $.317^{*}$ & \\
\hline 0 & 34 & $0.71 \pm 1.24$ & & & \\
\hline 1 disease & 14 & $0.36 \pm 0.93$ & & & \\
\hline$\geqq 2$ diseases & 22 & $1.0 \pm 1.38$ & & & \\
\hline Previous experience of motion sickness & & & 0.54 & $.590^{\#}$ & \\
\hline No & 54 & $0.69 \pm 1.24$ & & & \\
\hline Yes & 16 & $0.88 \pm 1.26$ & & & \\
\hline Previous experience of alcohol drinking & & & 0.86 & $.395^{\#}$ & \\
\hline No & 39 & $0.62 \pm 1.25$ & & & \\
\hline Yes & 31 & $0.87 \pm 1.23$ & & & \\
\hline Number of chemotherapy cycle & & & 4.96 & $.010^{*}$ & $3>1,2$ \\
\hline Second to third ${ }^{1}$ & 36 & $0.58 \pm 1.18$ & & & \\
\hline Fourth to fifth ${ }^{2}$ & 16 & $0.25 \pm 0.68$ & & & \\
\hline Sixth or more ${ }^{3}$ & 18 & $1.44 \pm 1.46$ & & & \\
\hline Additional use of antiemetics & & & 1.85 & $.069^{\#}$ & \\
\hline No & 67 & $0.67 \pm 1.19$ & & & \\
\hline Yes & 3 & $2.0 \pm 2.0$ & & & \\
\hline
\end{tabular}

* Analysis was performed using One-way ANOVA; ${ }^{*}$ Analysis was performed using independent samples $t$-test

\subsection{Implications for practice}

The study findings showed that the lung cancer patients with previous experience of motion sickness had higher level of nausea and that those receiving the sixth or more cycles of chemotherapy had higher level of vomiting during chemotherapy. Therefore, nurses should provide appropriate pre-chemotherapy nursing interventions such as increasing the use of antiemetics, non-pharmacological interventions (acupressure, massage, music therapy or relaxation) for lung cancer patients with who had received at least one cycle of Cisplatin $\left(50-75 \mathrm{mg} / \mathrm{m}^{2}\right)$, previous experience of motion sick- ness or those receiving the sixth or more cycles of chemotherapy to prevent the occurrence of nausea and vomiting.

\subsection{Limitations}

This study has some limitations that must be acknowledged. Due to time constraints and manpower limitations, this study only investigated lung cancer patients who intended to receive Cisplatin $\left(50-75 \mathrm{mg} / \mathrm{m}^{2}\right)$ in this hospitalization and explored the correlations between demographics and nausea/vomiting. Therefore, the generalizability of the study findings was limited. All of the participants in this study 
were lung cancer patients, thus it is difficult to generalize these result to other cancer patients. Future research should include more participants and extend more disease to better understand the correlations between demographics and nausea/vomiting. Therefore, future research should include biomarkers such as blood pressure, heart rate and respiratory rate to better provide evidence-based information.

\section{ACKNOWLEDGements}

This study was supported by a grant from the Kaohsiung Medical University Hospital (KMUH101-1M75).

\section{CONFlicts OF InTEREST Disclosure}

The authors have no conflicts of interest to disclose.

\section{REFERENCES}

[1] Ettinger DS, Akerley W, Borghaei H, et al. Non-small cell lung cancer. Journal of the National Comprehensive Cancer Network. 2013; 11(6): 645-53.

[2] Basch E, Prestrud AA, Hesketh PJ, et al. Antiemetics: American Society of Clinical Oncology clinical practice guideline update. Journal of clinical oncology: official journal of the American Society of Clinical Oncology. 2011; 29(31): 4189-98. http: //dx.doi.org/10.1200/JC0.2010.34.4614

[3] Ettinger DS, Armstrong DK, Barbour S, et al. Antiemesis. Journal of the National Comprehensive Cancer Network. 2012; 10(4): 456-85.

[4] Chou HL, Yao CT, Yang L, et al. Fatigue, Sleep Disturbances, and Mood Distress in Patients With Lung Cancer During Chemotherapy. Oncology Nursing. 2010; 10(2): 23-38.

[5] Janelsins MC, Tejani MA, Kamen C, et al. Current pharmacotherapy for chemotherapy-induced nausea and vomiting in cancer patients. Expert opinion on pharmacotherapy. 2013; 14(6): 757-66. http://dx.doi.org/10.1517/14656566.2013.776541

[6] Kamen C, Tejani MA, Chandwani K, et al. Anticipatory nausea and vomiting due to chemotherapy. European journal of pharmacology. 2014; 722: 172-9. http://dx.doi.org/10.1016/j.ejphar. 20 13.09 .071

[7] Roila F, Herrstedt J, Aapro M, et al. Guideline update for MASCC and ESMO in the prevention of chemotherapy- and radiotherapyinduced nausea and vomiting: results of the Perugia consensus conference. Annals of oncology: official journal of the European Society for Medical Oncology/ESMO. 2010; 21 Suppl 5: v232-43. http://dx.doi.org/10.1093/annonc/mdq194

[8] Basch E, Prestrud AA, Hesketh PJ, et al. Antiemetic Use in Oncology: Updated Guideline Recommendations from ASCO. American Society of Clinical Oncology educational book/ASCO American Society of Clinical Oncology Meeting. 2012: 532-40.

[9] Celio L, Agustoni F, Ricchini F, et al. Palonosetron plus dexamethasone in highly emetogenic chemotherapy: pooled data from two Phase III trials. Future oncology. 2013; 9(10): 1451-8. http: $/ /$ dx.doi.org/10.2217/fon.13.153

[10] Hilarius DL, Kloeg PH, van der Wall E, et al. Chemotherapy-induced nausea and vomiting in daily clinical practice: a community hospitalbased study. Supportive care in cancer: official journal of the Multinational Association of Supportive Care in Cancer. 2012; 20(1): 107-17. http://dx.doi.org/10.1007/s00520-010-1073-9

[11] Dewan P, Singhal S, Harit D. Management of chemotherapy-induced nausea and vomiting. Indian pediatrics. 2010; 47(2): 149-55.
[12] Grunberg S. Patient-centered management of chemotherapy-induced nausea and vomiting. Cancer control: journal of the Moffitt Cancer Center. 2012; 19(2 Suppl): 10-5.

[13] Shih V, Wan HS, Chan A. Clinical predictors of chemotherapyinduced nausea and vomiting in breast cancer patients receiving adjuvant doxorubicin and cyclophosphamide. The Annals of pharmacotherapy. 2009; 43(3): 444-52. http://dx.doi .org/10.1345/a ph. $1 \mathrm{~L} 437$

[14] Polit DF, Beck CT. Nursing research: generating and assessing evidence for nursing practice (9th ed.). 2012.

[15] Morrow GR. A patient report measure for the quantification of chemotherapy induced nausea and emesis: psychometric properties of the Morrow assessment of nausea and emesis (MANE). The British journal of cancer Supplement. 1992; 19: S72-4.

[16] Morrow GR. Methodology in behavioral and psychosocial cancer research. The assessment of nausea and vomiting. Past problems, current issues and suggestions for future research. Cancer. 1984; 53(10 Suppl): 2267-80.

[17] Hsieh LF, Liu CH, Chiu TC, et al. Clinical guideline for nonpharmacologic interventions pertaining to chemotherapy-induced nausea and vomiting among cancer patients. VGH Nursing. 2012; 29(2): 131-44.

[18] Rao KV, Faso A. Chemotherapy-induced nausea and vomiting: optimizing prevention and management. American health \& drug benefits. 2012; 5(4): 232-40

[19] Aapro M, Molassiotis A, Dicato M, et al. The effect of guidelineconsistent antiemetic therapy on chemotherapy-induced nausea and vomiting (CINV): the Pan European Emesis Registry (PEER). Annals of oncology: official journal of the European Society for Medical Oncology/ESMO. 2012; 23(8): 1986-92. http://dx.doi .org/10. 1093/annonc/mds021

[20] Jones JM, Qin R, Bardia A, et al. Antiemetics for chemotherapyinduced nausea and vomiting occurring despite prophylactic antiemetic therapy. Journal of palliative medicine. 2011; 14(7): 810-4. http://dx.doi.org/10.1089/jpm.2011.0058

[21] Chou FH. Symptom Assessment and Management of PregnancyRelated Nausea and Vomiting. Journal of Nursing. 2013; 60(6): 5-10.

[22] Liu CL, Guo JC, Xun YH. Progress of Constitutional Theory in Traditional Chinese Medicine. Zhejiang University of Traditional Chinese Medicine. 2009; 33(1): 138-9.

[23] Wang J, Li Y, Ni C, et al. Cognition research and constitutional classification in Chinese medicine. The American journal of Chinese medicine. 2011; 39(4): 651-60. http://dx.doi.org/10.1142/S $0192415 \times 11009093$ 